\title{
An XUV Optics Beamline at BESSY II
}

\author{
A.A. Sokolov*a, F. Eggenstein ${ }^{a}$, A. Erko ${ }^{a}$, R. Follath ${ }^{b}$, S. Künstner ${ }^{a}$, M. Mast ${ }^{a}$, J.S. Schmidt ${ }^{a}$, F.

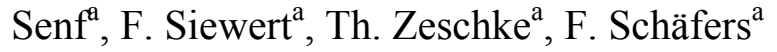 \\ ${ }^{a}$ Institute for Nanometer Optics and Technology, Helmholtz-Zentrum Berlin, Albert-Einstein-Str. 15, \\ Berlin, Germany, D-12489; \\ ${ }^{\mathrm{b}}$ Paul Scherrer Institut, Villigen PSI, Switzerland, 5232 \\ *Corresponding author, email: andrey.sokolov@helmholtz-berlin.de
}

\begin{abstract}
The design for a new XUV-Optics Beamline is presented. The collimated plane grating monochromator (PGM-) beamline at a bending magnet is setup at the BESSY-II synchrotron radiation facility within the framework of the blazed-grating production facility. Coupled to a versatile four-circle (ten axes) UHV- reflectometer as a permanent end station the whole setup is dedicated to at-wavelength characterization and calibration of the in-house produced precision gratings and novel nano-optical devices as well as mirrors, multilayered systems etc. It is also open to external projects employing reflectometry, spectroscopy or scattering techniques. According to its purpose, this beamline has specific features, such as: very high spectral purity, provided by two independent high order suppression systems, an advanced aperture system for suppression of stray light and scattered radiation, a broad energy range between $10 \mathrm{eV}$ and $2000 \mathrm{eV}$, small beam divergence and spot size on the sample. Thus this Optics Beamline will become a powerful metrology tool for reflectivity measurements in s- or p-polarisation geometry with linearly or elliptically polarized light on real optics up to $360 \mathrm{~mm}$ length and $4 \mathrm{~kg}$ weight.
\end{abstract}

Keywords: At-wavelength metrology, UV, EUV, VUV, XUV, reflection gratings, reflectometry, polarimetry, synchrotron instrumentation

\section{INTRODUCTION}

Since the takeover of the grating production equipment from ZEISS company three years ago, blazed and laminar gratings are successfully produced in our Institute for Nanometre Optics and Technology (INT) at the BESSY-II synchrotron radiation facility [1]. Some of these gratings have been delivered to other SR-facilities already [2]. Other focusing and dispersing nano-optical devices such as arrays of variable period reflective zone plates (RZP) [3-5] are also produced in-house. For characterization and metrology of these elements the INT has a powerful laboratory for ex-situ and in-situ metrology. Information about surface quality and finish of the optical elements (roughness; slope; curvature; shape, line density, profile depth of nanostructures and their defects) are collected from (1) the Nanometre Optics Slope Measuring Machine (NOM) [6, 7], (2) Atomic Force Microscopy (AFM), (3) White Light Interferometry (WLI) and (4) $\mathrm{X}$-ray Diffractometry (XRD) [8]. Such characterization is very useful and extremely important during the development and production process. It is used to iteratively influence, modify and optimize the production parameters as well as to simulate the expected performance of an X-ray optical system based on real topography data.

The final test drive, however, is the at-wavelength metrology to determine the reflectivity or the diffraction efficiency of the investigated optical structures at the wavelength of interest. Currently at BESSY-II two experimental stations, a reflectometer [9] and a polarimeter [10] are in operation which are used since many years for at-wavelength reflectometry [11, 12], polarimetry [13,14] and ellipsometry [15] measurements: However, the polarimeter is designed for small-sized test samples only, not suitable for real optics. The reflectometer operates with larger samples, but has other limitations and no in-situ sample adjustment is possible. It is mounted at the intermediate focus of the old optics beamline PM-4 [9] which is in shared use with another spectroscopy experiment after the refocussing section. Thus access to the reflectometer vacuum system is limited and leaves no flexibility to experimental modifications. 
Apart from at-wavelength metrology for x-ray optical elements, reflectometry is also a very powerful scientific technique. It allows to carry out nondestructive characterization and depth-profiling of microstructures, layered systems and burred interlayers [16, 17]. It enables to reconstruct atomic concentration profiles [18, 19], to investigate polarization dependence and anisotropy effects of helical substances [20,21] or to get information about roughness of surface and buried interface structures [22]. Based on accurate reflection coefficient spectra it is possible to calculate the spectral dependence of optical constants [23, 24].

The grating project enabled us to setup a new Optics Beamline and a new Reflectometer as permanent end station, which are exclusively dedicated to optical metrology at short-term request. It is open to outside users. It is a significant improvement of the existing setup and addresses all requirements for quantitative reflectometry as known from other laboratories [25-27]:

- flexible operation of PGM in high resolution, high flux or high-order suppression modes

- highest spectral purity from higher diffraction orders

- low divergence of incident beam

- aperture system for suppression of scattered and stray light

- large working energy range with appropriate energy resolution

- $\quad \mathrm{s}$ - and p-polarisation geometry

- option for elliptically polarized light operation (polarimetry, ellipsometry)

- six degrees-of-freedom sample alignment system

- wide angular range for specular and off-specular scans

- wide dynamic range detectors with /without slits

- maximum sample size up to $360 \mathrm{~mm}$ length and $4 \mathrm{~kg}$

In this paper we discuss the design of the Optics Beamline, its main technical features and performance calculations including raytracing. Information about the Reflectometer endstation for this beamline is given elsewhere [28] and within these proceedings [29].

\section{GENERAL LAYOUT}

The New Optics Beamline is presently set up at the BESSY II dipole section DIP 1.1. The source parameters are listed in Table 1. Figure 1 presents the beamline optical layout. It is a plane grating monochromator (PGM) with a variable plane mirror operated in collimated beam [30]. The beamline acceptance determined by the dimension and incidence angle of the first mirror is $0.5 \times 2.33 \mathrm{mrad}^{2}(\mathrm{v} \times \mathrm{h})$. Mirror M1 focusses the incident beam horizontally in front of the HiOS chamber and collimates vertically. As monochromator we use an existing SX-700 (PM-1 of BESSY-I) [31, 32] equipped with two plane gratings and with a rotatable plane mirror M2. The monochromator operation in collimated light enables a very flexible operation at different $\mathrm{c}_{\mathrm{ff}}$-values or on-blaze [30]. The beam is then vertically focused by the cylindrical mirror M3 onto the exit slit (SL). The refocusing toroidal mirror M4 focuses the light onto the sample position. As a final conditioning step the beam passes through a filter and slit unit (FSU), a double set of slits and absorption filters and an intensity monitoring system in front of the reflectometer experimental station. All focusing optical elements of the beamline are listed in Table 2, the monochromator optics is listed in Table 3.

During the design process the beamline was intensively simulated with the raytracing program RAY [34]. After delivery of the mirrors the simulations have been repeated with their real parameters and measured slope and finish errors, and the beamline design was adjusted adequately. Figure 2 presents the calculated beam size across the length of the beamline in vertical and horizontal planes. These calculations clearly show the beam divergence, size and flux density at any part of the beamline. 
Table 1. BESSY II source characteristics of the dipole section DIP 1.1

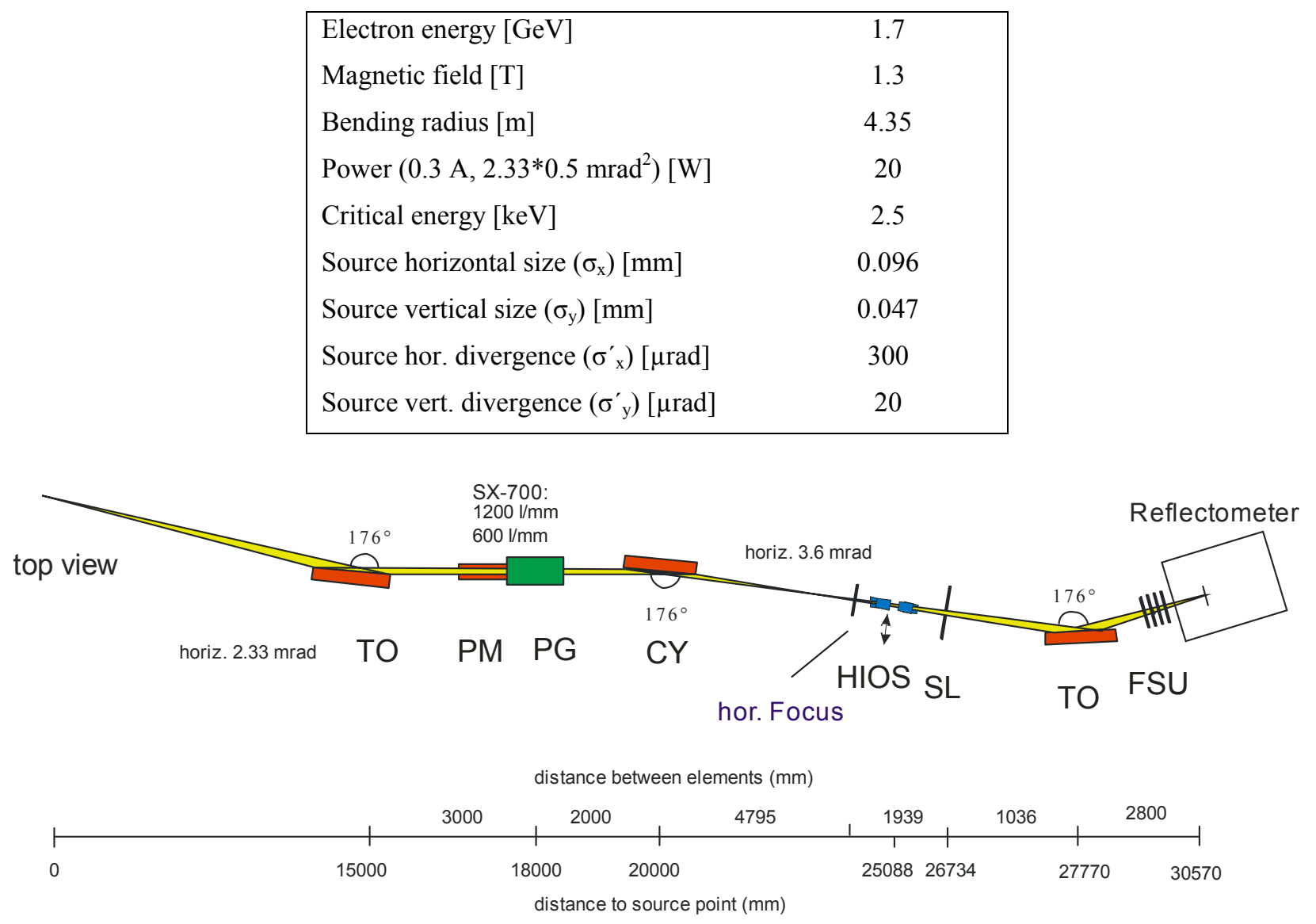

Figure 1. Optical layout of the Optics Beamline as seen from top.

The beamline can also be operated with off-plane radiation from the BESSY-II bending magnet. Thus the polarization can be changed from horizontal linear polarization $(\mathrm{S} 1=+1)$ to elliptical polarization with a selectable degree of circular polarization ( $\mathrm{S} 3<=+/-0.8$ ) [35]. This is done by an azimuthal rotation of the premirror M1 accompanied by an equal but opposite change of incidence angle of the monochromator mirror M2. Thus the incidence angle on the grating is unaffected by this operation and no energy shift is encountered during change of polarization.

Table 2. Parameters of the focusing mirrors of the beamline

\begin{tabular}{|lccc|}
\hline Optical element & M1 & M3 & M4 \\
\hline Shape & toroidal & cylindrical & toroidal \\
Total surface size $(\mathrm{L} \mathrm{x} \mathrm{W)}[\mathrm{mm}]$ & $1000 * 60$ & $1000 * 60$ & $350 * 30$ \\
Material & $\mathrm{Au}(40 \mathrm{~nm}) / \mathrm{Si}$ & $\mathrm{Au}(40 \mathrm{~nm}) / \mathrm{Si}$ & $\mathrm{Au}(40 \mathrm{~nm}) / \mathrm{Si}$ \\
Long radius R $[\mathrm{mm}]$ & 339578 & 194000 & 82725 \\
Short radius $\rho[\mathrm{mm}]$ & 1040.3 & 470.3 & 52.8 \\
Angle of incidence $[$ deg.] & $2^{\circ}$ & $2^{\circ}$ & $2^{\circ}$ \\
Source distance $(\mathrm{h} / \mathrm{v})[\mathrm{mm}]$ & 15000 & $-/ \infty$ & $2975 / 1036$ \\
\hline
\end{tabular}




\begin{tabular}{|lccc|}
\hline Focus distance $(\mathrm{h} / \mathrm{v})[\mathrm{mm}]$ & $9795 / \infty$ & $-/ 6734$ & 2800 \\
Roughness $\sigma(\mathrm{rms})[\mathrm{nm}]$ & 0.25 & 0.23 & 0.25 \\
Slope error $(\mathrm{mer} . / \mathrm{sag}).[\operatorname{arcsec} \mathrm{rms}]$ & $0.17 / 0.65$ & $0.18 / 0.7$ & $0.3 / 1.0$ \\
\hline
\end{tabular}
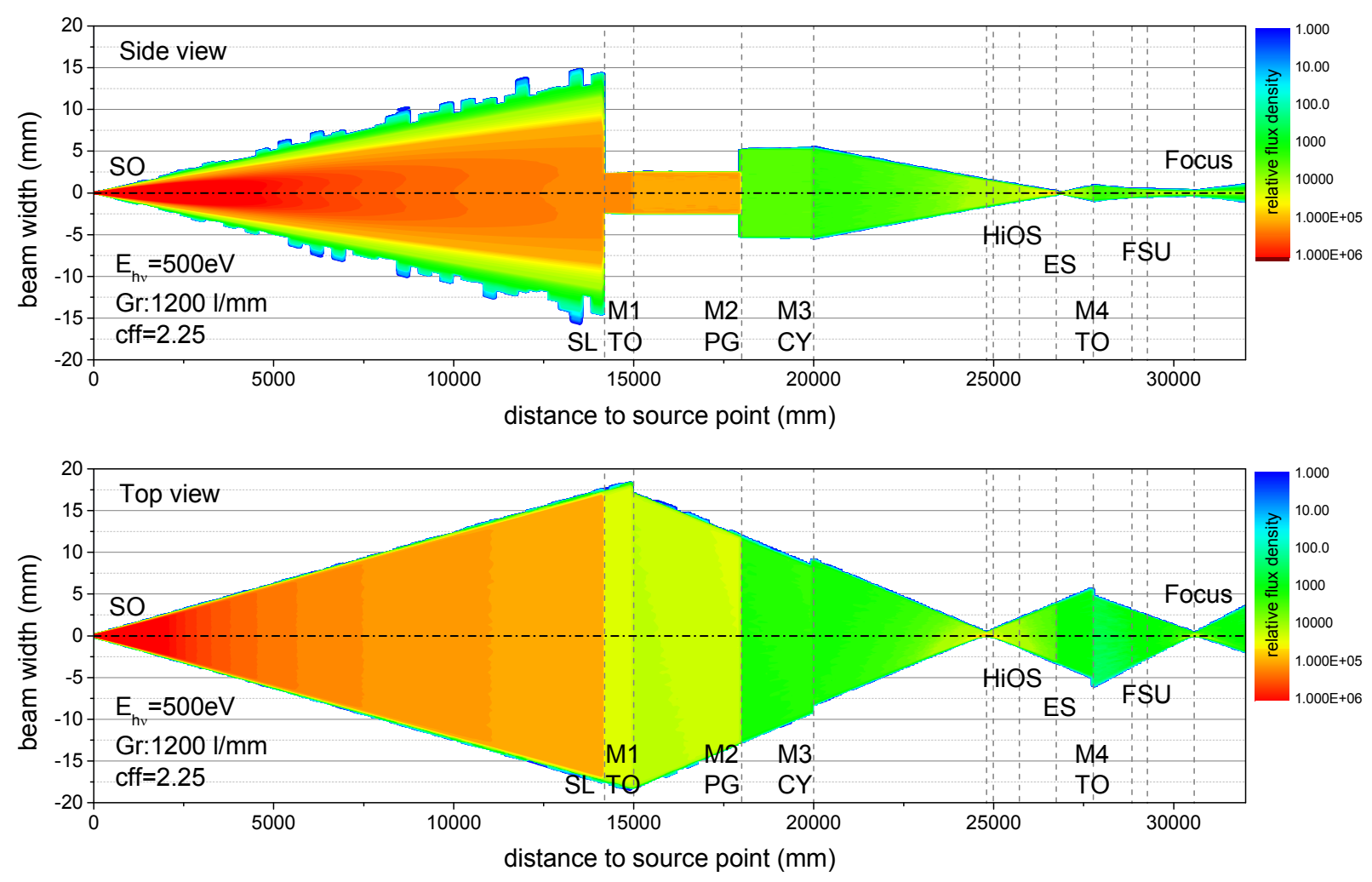

Figure 2. Beam size in vertical and horizontal plane along the beamline for typical operation parameters

Table 3. Parameters of the SX-700 monochromator optics

\begin{tabular}{|lccc|}
\hline Optical element & PG1 & PG2 & M2 \\
\hline Shape & plane grating & plane grating & plane mirror \\
Optical surface size $[\mathrm{L} \mathrm{x} \mathrm{W,} \mathrm{mm]}$ & $120 \times 40$ & $120 \times 40$ & $600 \times 40$ \\
Material & $\mathrm{Au}(40 \mathrm{~nm}) / \mathrm{Si}$ & $\mathrm{Au}(40 \mathrm{~nm}) / \mathrm{Si}$ & $\mathrm{Au}(40 \mathrm{~nm}) / \mathrm{Zerodur}$ \\
Long radius $[\mathrm{km}]$ & 95 & 94 & $>300$ \\
Line density $[1 / \mathrm{mm}]$ & 1200 & 600 & - \\
Blaze angle $[$ deg.] & $1.1^{\circ}$ & $2^{\circ}$ & - \\
Angle of incidence $[\mathrm{deg}]$. & $1^{\circ}-24^{\circ}$ & $1^{\circ}-24^{\circ}$ & $1.5^{\circ}-13^{\circ}$ \\
Roughness $\sigma(\mathrm{rms})[\mathrm{nm}]$ & 0.3 & 0.6 & 0.5 \\
Slope error $[$ arcsec rms] & 0.04 & 0.06 & 0.06 \\
Fabricated by & HZB & HZB & ZEISS \\
\hline
\end{tabular}




\section{FLUX AND RESOLUTION}

The photon flux and energy resolution of the optics beamline are presented in figure $3(a, b)$ and figure 4 as calculated using RAY [34]. Shown are the results for the $1200 \mathrm{l} / \mathrm{mm}$ grating at different $\mathrm{c}_{\mathrm{ff}}$ values, which is defined as the ratio between the cosines of the diffracted angle $\beta$ to the incident angle $\alpha$ on the grating for the particular diffraction order $\mathrm{m}$ ( $c>1$ : inside order, $m>0, \alpha>\beta, c<1$ : outside order, $m<0, \beta>\alpha$ ). The figures demonstrate the beamline flexibility between high flux and high resolution operation. At standard setting $\mathrm{c}_{\mathrm{ff}}=2.25$ and $100 \mu \mathrm{m}$ exit slit we expect a photon flux of $10^{10}$ $-10^{11}$ photons $/ \mathrm{s} / 100 \mathrm{~mA}$ at a moderate resolution of $\mathrm{E} / \Delta \mathrm{E}=2000-5000$. This moderate resolution is adequate for atwavelength metrology and reflectometry experiments for which this beamline has been designed. Energy calibration and resolution tests can routinely be performed by absorption spectroscopy on suitable gases $\left(\mathrm{Ar}, \mathrm{Kr}, \mathrm{Ne}, \mathrm{N}_{2}\right)$ with an ionization chamber mounted permanently between the exit slit and the refocussing mirror.
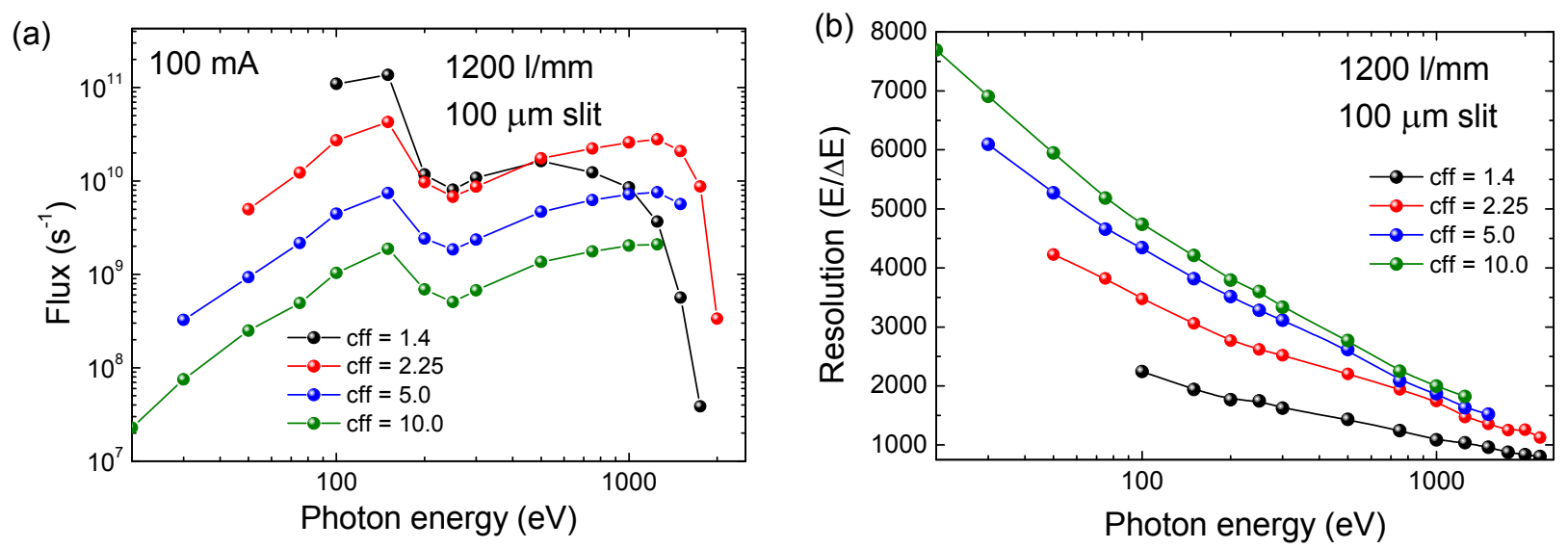

Figure 3. Photon flux (a) and energy resolution (b) calculated with RAY for the $1200 \mathrm{l} / \mathrm{mm}$ grating at different $\mathrm{c}_{\mathrm{ff}}$ values.

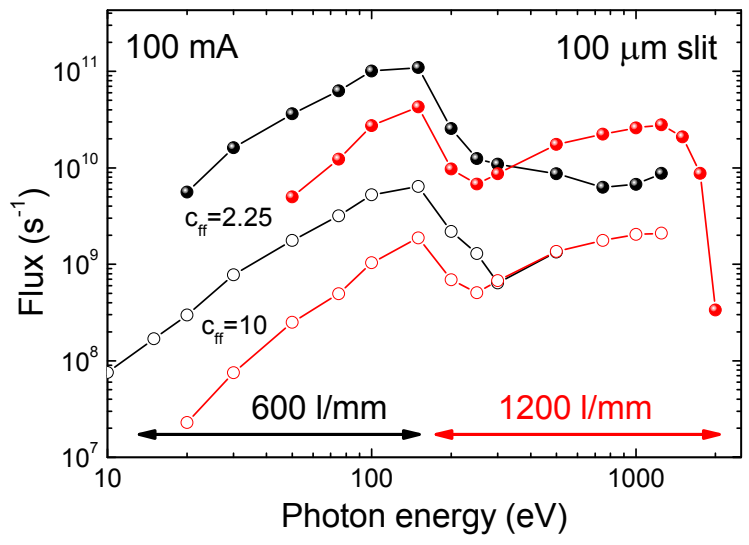

Figure 4. Photon flux calculated with RAY for the $600 \mathrm{l} / \mathrm{mm}$ and $1200 \mathrm{l} / \mathrm{mm}$ gratings at $\mathrm{c}_{\mathrm{ff}}$ values of 2.25 (solid circles) and 10 (open circles).

\section{BEAM SIZE AND DIVERGENCE AT SAMPLE}

Standard reflectometry requires beam sizes large enough to collect information with good statistics across the sample surface. But it should also be adjustable to have sufficient spatial resolution for investigation of small structures like reflection zone plates or when scanning a sample across the surface. Our beamline will have a balance of these two options and the average spot size will stay in the limits of $0.2 \times 0.3 \mathrm{~mm}^{2}(\mathrm{v} \times \mathrm{h})$. As example, in figure 5 the relative flux 
density map of the beam at the sample in the reflectometer chamber is shown. Standard beamline settings as given in the figure have been used here. The beamline settings have a strong effect on the spot size. It is influenced by the exit slit width, beamline acceptance slits in front of the M1 mirror, $\mathrm{c}_{\mathrm{ff}}$ factor of the monochromator and the set of slits and apertures in the FSU chamber.

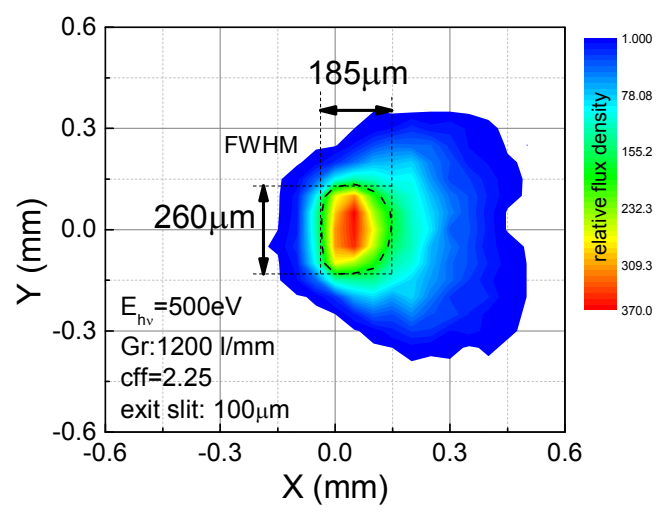

Figure 5. Relative flux density at the reflectometer sample position. FWHM beam size is $260 \mu \mathrm{m} \times 185 \mu \mathrm{m}$ with the indicated beamline settings.
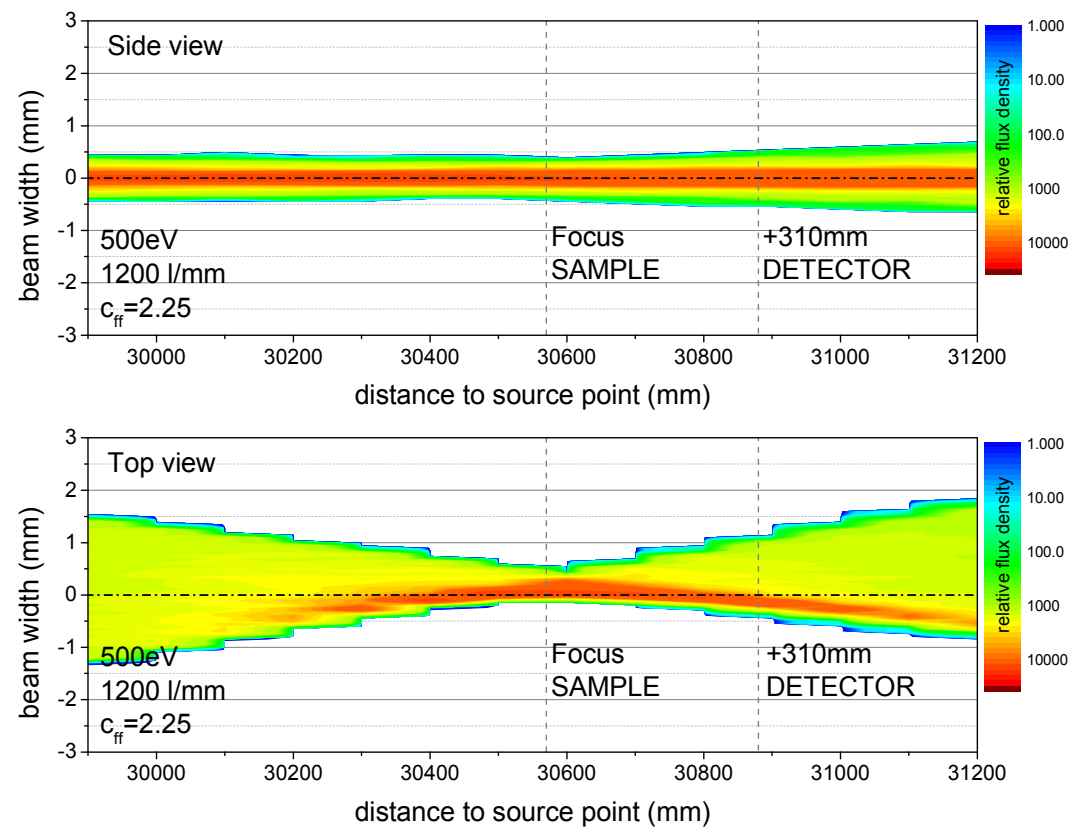

Figure 6. Beam divergence at the focus position in horizontal and vertical plane.

In Figure 6 the divergence of the beam in the vicinity of the focus is shown. It is $0.5 \mathrm{mrad} \times 3.6 \mathrm{mrad}(\mathrm{v} \mathrm{x} \mathrm{h})$. For the reflectometer operating in the vertical reflection plane (employing s-polarisation geometry) this is sufficiently collimated and it allows to neglect the increase of beam size up to the detector $250 \mathrm{~mm}$ further downstream. All the specular reflected beam and most part of the scattered radiation is well within the acceptance area of $4 \mathrm{x} 4 \mathrm{~mm}^{2}$ of the photodiode when the detector is used without apertures to define angular resolution. 


\section{WORKING ENERGY RANGE}

The optics beamline is equipped with an SX-700 plane grating monochromator which had been in use from 1981 to 1999 at the BESSY-I storage ring as Petersen-Monochromator (PM-1) [31, 32]. For the new operation the control was upgraded to the BESSY-II VME-based standard beamline control system under EPICS. The two 30 year old gratings have been replaced by new ones produced in-house with state of the art residual slope errors of 0.05 arcsec rms, see also table 3 and [33]. The original M2 plane mirror of $600 \mathrm{~mm}$ length was passed through a cleaning process before monochromator alignment. Then the monochromator was finally adjusted, aligned and the movements of mirror and grating were extensively calibrated. The Heidenhain position control of the motorized sine bar travel outside vacuum was calibrated against the angular scale ( $\theta$ mirror incidence angle, $\alpha$ and $\beta$ grating incidence and diffraction angle) using an electronic autocollimator which measured the parallel scan of mirror and grating over the whole angular range on an accuracy level of 1 arc sec. The horizontal deviation of the beam position across the full angular range of mirror and grating is less than $30 \mu \mathrm{rad}$.

One of the advantages of the SX-700 is the wide angular range from $1.5^{\circ}$ to $24^{\circ}$ for the grating and from $1.5^{\circ}$ to $13^{\circ}$ for mirror. This transfers into a wide operating energy range without grating change. Figure 7 presents the relative beamline transmission for both gratings as function of energy and $\mathrm{c}_{\mathrm{ff}}$-factor. Thus these plots determine the available energy range which depends on the $c_{\mathrm{ff}}$-factor. It spans from $20 \mathrm{eV}$ to $2200 \mathrm{eV}$ at standard $\mathrm{c}_{\mathrm{ff}}$ factor (2.25) using both gratings. The range can be extended down to $10 \mathrm{eV}$ for larger $\mathrm{c}_{\mathrm{ff}}$ at the price of spectral purity.
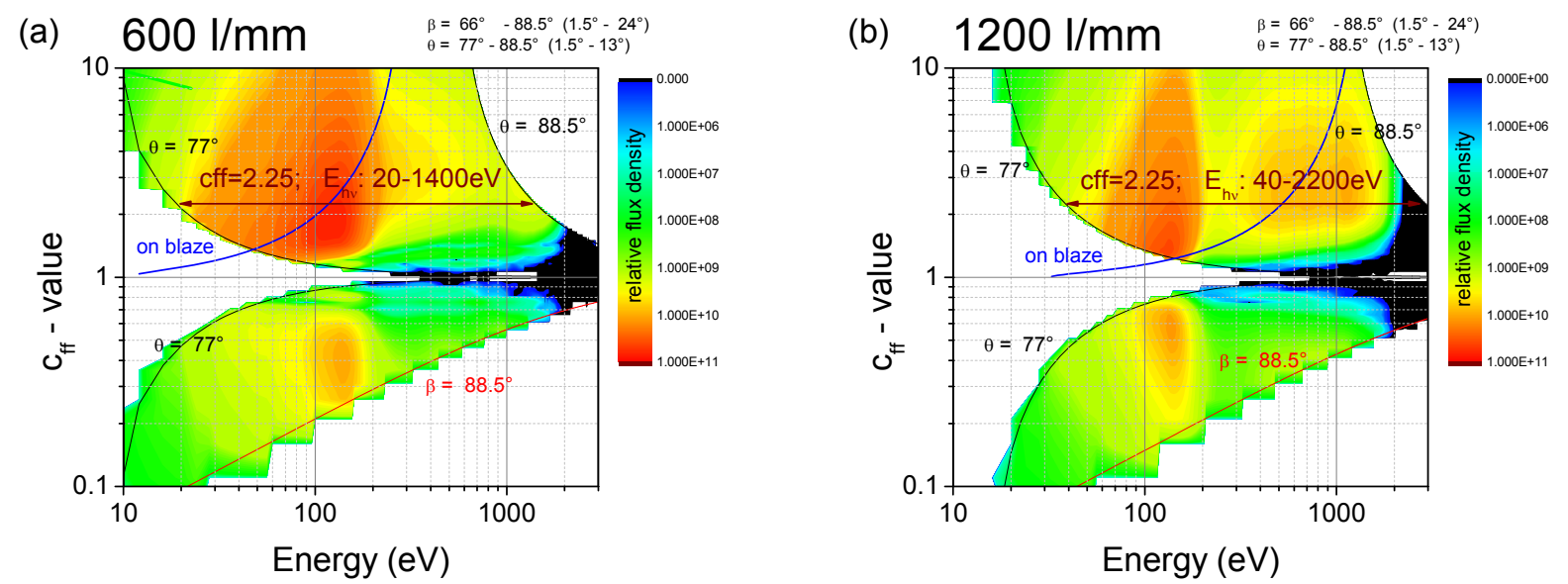

Figure 7. Relative flux densities for (a) - 6001/mm and (b) - 12001/mm grating calculated with REFLEC [36] for the available angular (energy) and $\mathrm{c}_{\mathrm{ff}}$-range of the SX-700 monochromator. Blue curve represents an on-blaze scan.

\section{SPECTRAL PURITY}

The quality of a reflectometry or metrology setup is strongly dependent on the accuracy of the measured absolute values of the reflection coefficient, which should be on a sub-percent level. One of the strongest requirements for this is the spectral purity of the incident radiation. Presence of high diffraction orders in the beam have a detrimental effect on the measured reflectivity. In general determined reflection coefficient $(R=I / I o)$ determined in sample-in $(I) /$ sample-out $(I o)$ technique will be reduced. Figure 8 shows the calculation of $2^{\text {nd }}$ order and $3^{\text {rd }}$ order transmission, respectively, for the $1200 \mathrm{l} / \mathrm{mm}$ grating as function of $\mathrm{c}_{\mathrm{ff}}$ and energy. For $\mathrm{c}>1$ (inside order, $\alpha>\beta$ ) high orders can reach a level of more than $10 \%$ for $2^{\text {nd }}$ order or $1 \%$ for $3^{\text {rd }}$ order. The lower line density grating $(6001 / \mathrm{mm})$ has higher efficiency (figure 7$)$ but also higher amount of higher order. Usually most problematic is the low energy range because here even the 4th, 5th and even higher orders can also be significant. Standard high-order suppression by reducing $\mathrm{c}_{\mathrm{ff}}$ to the lowest possible value does not hold here, since this range is accessible only at high $\mathrm{c}_{\mathrm{ff}}$. 

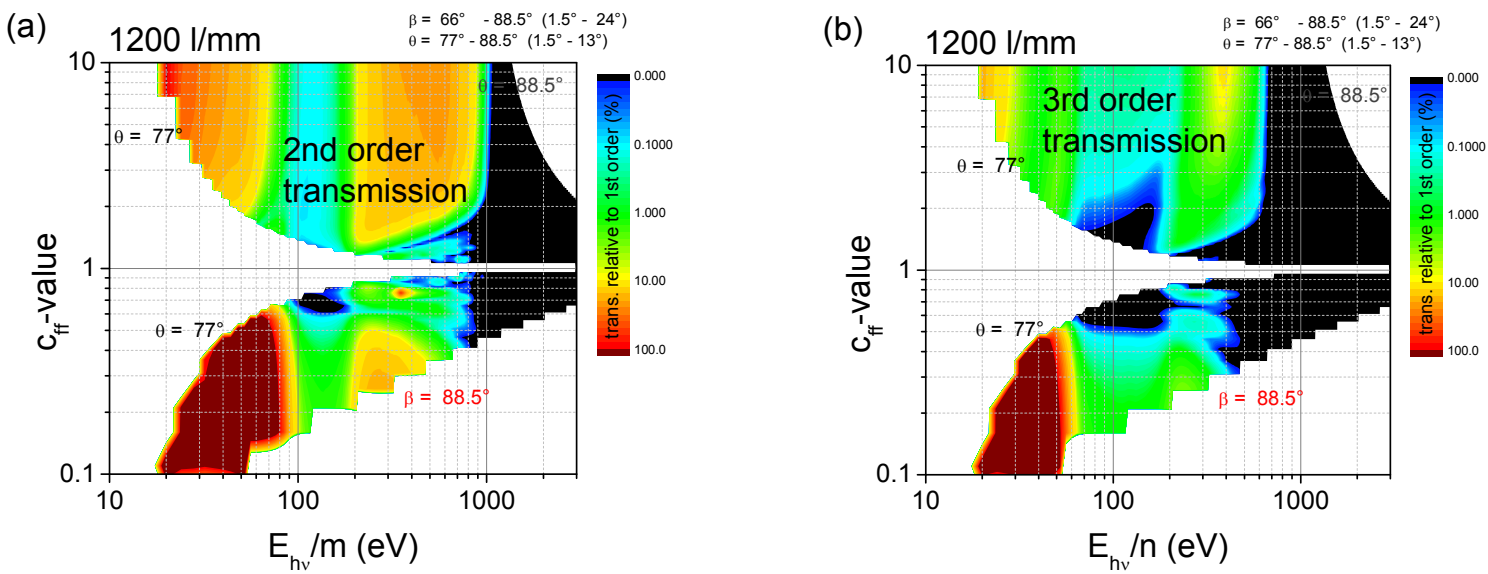

Figure 8. Transmission of second (a) and third (b) order, respectively, normalized to first order transmission for the 1200 $1 / \mathrm{mm}$ grating as function of energy and $\mathrm{c}_{\mathrm{ff}}$-factor. The energy scale is given for $\mathrm{m}=1 \mathrm{st}$ order.

For effective high order suppression the optics beamline is equipped with two additional systems for effective high order suppression insertable into the beam path. The first one is a double set of absorber filters installed in a specially designed filter-slit unit chamber (FSU) between refocussing mirror and reflectometer station. Exploiting the transmission cut-off at absorption edges photons with twice the energy of the first order may be suppressed by a factor of $10-1000$. Using a couple of different absorber materials the total energy range can be covered. The figure 9 shows the calculated transmission of first order and the suppression of second order for a variety of filter materials. To reach a balance between transmission and suppression efficiency most filter were calculated with a thickness of $750 \mathrm{~nm}$, except for Al $(500 \mathrm{~nm})$ and $\mathrm{C}_{6} \mathrm{H}_{8}(1500 \mathrm{~nm})$, respectively. The FSU chamber has 12 filter slots on two identical feedthroughs with standard frames. Thus two filters can be inserted into the beam simultaneously, which can be either identical to double the suppression effect or of filter combinations to achieve even $3^{\text {rd }}$ or higher order suppression.

In the same FSU chamber two sets of interchangeable apertures can be inserted into the beam, one of them upstream, and the other downstream the filter feedthroughs. The size of these apertures allows for cut off scattered incident light as well as shaping the beam in one (horizontally by slits) or two directions (pinholes). The downstream aperture unit allows for suppressing scattering of the filters.

As additional option the filters are electrically isolated. Thus measurement of the drain current is possible for use as an in-situ intensity monitor (Io), and potentially, in combination with a small pinhole (smaller than the beam size) as beam position monitor.

The second high order suppression system (HiOS) is a four mirror system [37 - 39] as shown schematically in figure 10. The four mirrors are insertable into the beam, the first two and the last two mirrors, aligned parallel to each other on a common platform, are rotated by two goniometers in opposite directions to have no vertical offset of the reflected beam. The rotation axes of the goniometers lie in the center of the first and last mirror. Therefore the other mirrors 2 and 3 are longer and the beam glides along the surface while changing the incidence angle. Two sets of 4-mirror systems (System I and II) are mounted next to each other on the same translation stage with different vertical offset to realize different angular scan ranges as given in Table 4. The high energy cut-off is freely selectable by the incidence angle chosen, due to drop of reflectivity above the critical angle. This effect is strongly dependent on the optical constants and thus on the coating material. Figure 11 demonstrates the transmission and high order suppression capability for three coatings materials at different angles. In this way most of the energy range between $20 \mathrm{eV}$ and $700 \mathrm{eV}$ can be covered with a suppression efficiency of $10^{4}$ while keeping transmission at approximately $40 \%$. Thus a properly aligned HiOS is more effective than the filter unit. 


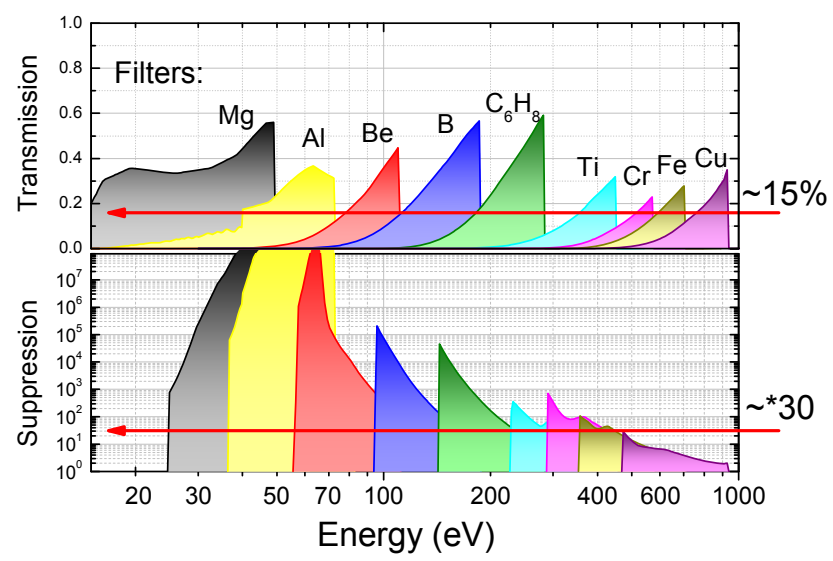

Figure 9. Calculated second order suppression and transmission for higher order suppression system by the set of absorber filters in the FSU chamber.

Table 4 Parameters of the HiOS mirror systems I and II

\begin{tabular}{|l|c|c|}
\hline High Order & $\begin{array}{c}\text { 4-mirror } \\
\text { System I }\end{array}$ & $\begin{array}{c}\text { 4-mirror } \\
\text { System II }\end{array}$ \\
\hline Mirror sizes (LxW) [mm] & $20 \times 20$ & $40 \times 40$ \\
M1, M4 & $120 \times 20$ & $180 \times 40$ \\
M2, M3 & 3 & 20 \\
Mirror offset [mm] & $2^{\circ}-12^{\circ}$ & $8^{\circ}-70^{\circ}$ \\
Angular range $\left[^{\circ}\right]$ & $\mathrm{Si}$ & $\mathrm{Si}$ \\
Substrate & --- & $\mathrm{C}(35 \mathrm{~nm})$ \\
Coatings (thickness $[\mathrm{nm}])$ & & $\mathrm{AlF}_{3}(35 \mathrm{~nm})$ \\
\hline
\end{tabular}




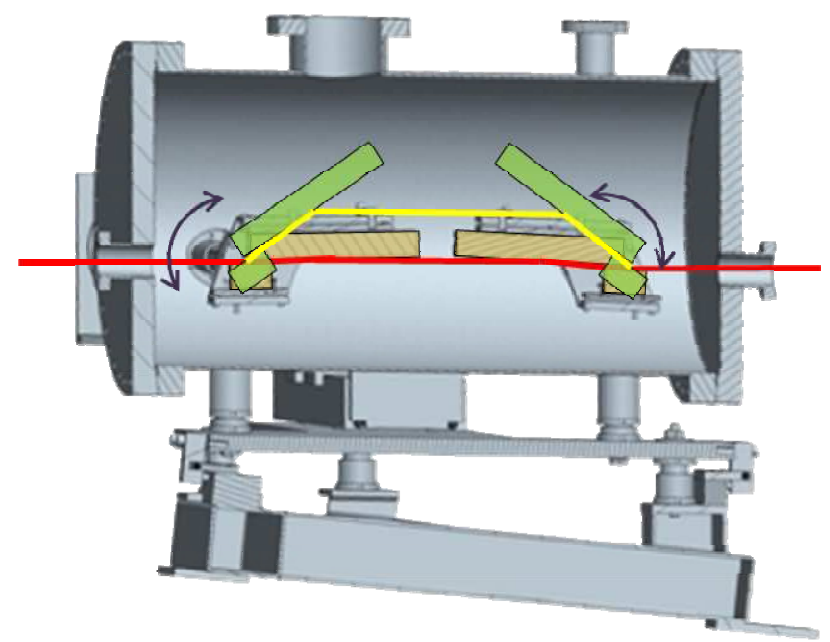

Figure 10. High Order Suppression system (HiOS) with two sets of four mirrors.

Thus the two high order suppression systems on the optics beamline provide a wide flexibility for light shaping upstream of the reflectometer. Absorption filters are easy to use and have a large $2^{\text {nd }}$ order suppression capability, but not for higher orders and at the price of increased scattered radiation. The HiOS system suppresses higher orders even more powerful, but it needs to be aligned very accurately in order not to change beam direction.

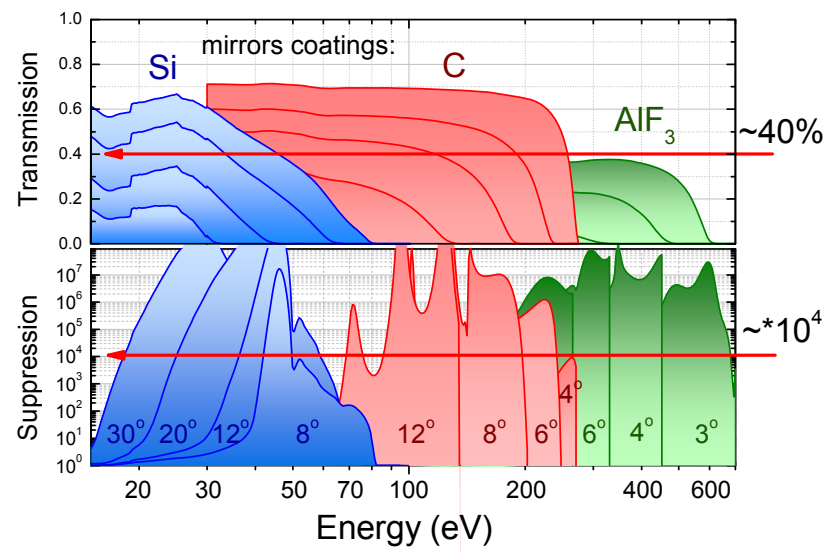

Figure 11. Calculated second suppression and transmission for the 4-mirror High order Supression System HiOS.

\section{REFLECTOMETER}

The UHV-reflectometer is described elsewhere [28] and in these proceedings [29]. This is a four circle goniometer (with two for sample scans and two for the detector) which is schematically shown in Figure 12. It was specially designed to be coupled to the Optics Beamline as a fixed end station. The vacuum vessel has a diameter of $1 \mathrm{~m}$ and it can handle large samples up to $360 \mathrm{~mm}$ length and $4 \mathrm{~kg}$ weight. Smaller samples (e.g. wafers) are mounted via a load lock system without breaking vacuum. The reflectometer houses 3 HUBER-goniometers (three circles) for setting the (1) sample's azimuthal angle (s- or p-polarisation, respectively), (2) sample's incidence angle $\theta\left(0^{\circ}-90^{\circ}\right)$ and (3) the detector arm $2 \theta$ $\left(0^{\circ}-180^{\circ}\right)$. For off-plane sample scans $\left(-4^{\circ}-+4^{\circ}\right)$ another motorized stage is hooked up on the $2 \theta$-goniometer. The sample adjustment and positioning system is based on a newly designed compact UHV-tripod system for six degrees of 
freedom (six axes) which is schematically shown in Figure 13. It allows a two-dimensional scan of the samples within a range of approximately $+/-20 \mathrm{~mm}$ while maintaining all rotational degrees of freedom within $+/-1^{\circ}$. We expect a pointing stability of this system of better than $10 \mu \mathrm{m}$ and $0.01^{\circ}$. A couple of photodiode detectors with and without pinholes or apertures are available.

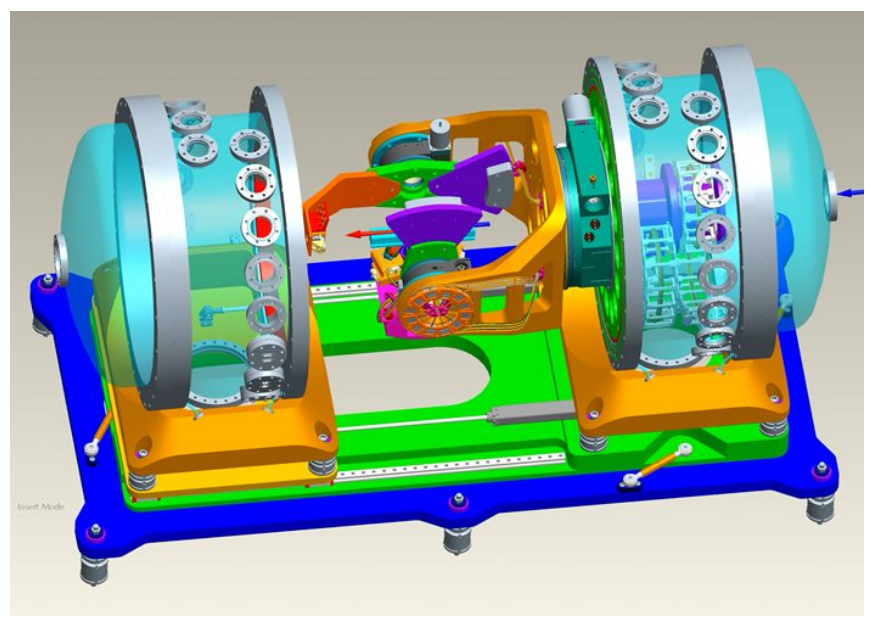

Figure 12. The four-circle (ten axes) UHV-Reflectometer chamber with the UHV-optical bench for sample and detector positioning.

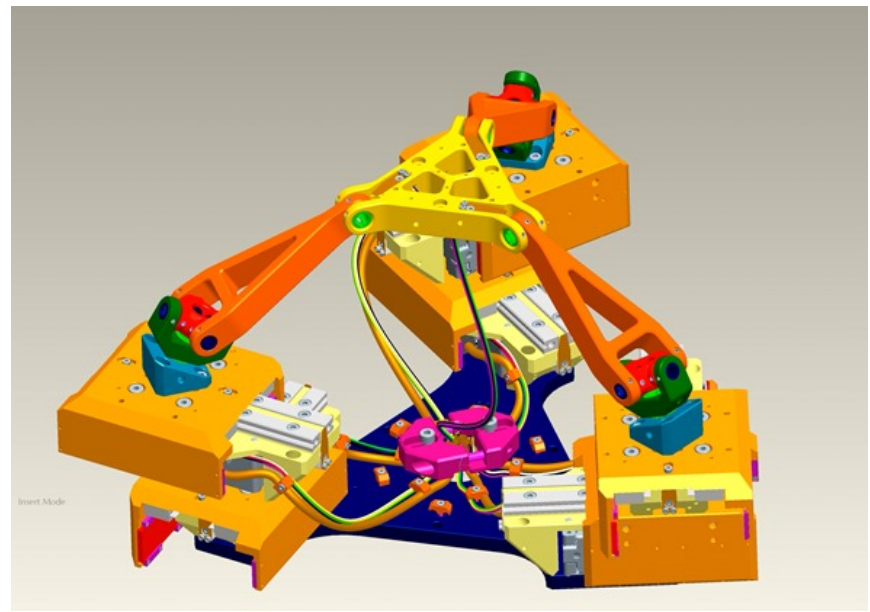

Figure 13. The UHV-tripod unit for sample adjustment in six degrees of freedom.

\section{SUMMARY}

The new Optics Beamline at BESSY-II in combination with a new Reflectometer as permanent end station for large samples will provide XUV radiation at high flexibility and in such a quality to allow for quantitative high-precision atwavelength metrology of various optical devices as well as for reflectometry technics for investigations within a wide range of scientific applications, such as novel nano-optical devices, multilayered XUV optics, depth-profiling of nanoelectronic structures and many others. The main demands for such kind of experiments are a wide photon energy range, low divergence and low scattering of the incident beam at moderate energy resolution. 
Presently the beamline and reflectometer are set into operation and commission will start this summer. The setup will also be open for external users at short term access.

\section{ACKNOWLEDGMENTS}

Financial support of the European Community is gratefully acknowledged (EFRE-contract no. 20072013 2/43).

\section{REFERENCES}

[1] Loechel, B., Erko, A., Lemke, St., Nelles, B., Schmidt, M. and Senf, F., "Installation of a technological center for highly efficient optical gratings at Helmholtz-Zentrum Berlin (HZB),” J. Phys.: Conf. Ser. 425, 212012 (2013).

[2] Lemke, S., Seliger, T., Rudolph, I., Kutz, O., Goettert, Ph., Nelles, B., Senf, F., Loechel, B., "Status of laminar grating manufacturing via lithography at HZB," Microsyst. Technol. 20, 1, (2014).

[3] Brzhezinskaya, M., Firsov, A., Holldack, K., Mitzner, R., Pontius, N., Schmidt, J.-S., Sperling, M., Stamm, C., Föhlisch, A., Erko, A., "A novel monochromator for experiments with ultra-short X-ray pulses," J. Synchrotron Rad. 20, 522-530 (2013).

[4] Könnecke, R., Follath, R., Pontius, N., Schlappa, J., Eggenstein, F., Zeschke, T., Bischoff, P., Schmidt, J.-S., Noll, T., Trabant, C., Schreck, S., Wernet, Ph., Eisebitt, S., et al., "The confocal plane grating spectrometer at BESSY II," J. Electron Spectrosc. Relat. Phenom. 188, 133-139 (2013).

[5] Erko A., Firsov A., Senf F., "Novel parallel vacuum ultra-violet/X-ray fluorescence spectrometer," Spectrochimica Acta Part, B 67, 57-63 (2012).

[6] Siewert, F., Buchheim, J., Höft, T., Fiedler, S., Bourenkov, G., Cianci, M., Signorato, R., "High angular resolution slope measuring deflectometry for the characterization of ultra-precise reflective x-ray optics," Meas. Sci. Technol. 23, 074015 (2012).

[7] Siewert, F., Buchheim, J., Höft, T., Zeschke, T., Schindler, A., Arnold, T., "Investigations on the spatial resolution of autocollimator-based slope measuring profilers," Nuclear Instruments and Methods in Physics Research A 710, 42-47 (2013).

[8] Erko, A., Abrosimov, N. V., Alex, V., "Laterally-Graded SiGe Crystals for High Resolution Synchrotron Optics," Crystal Research and Technology, 37, 685-704 (2002).

[9] Schäfers, F. Cimino, R., "Soft X-ray reflectivity: from quasi-perfect mirrors to accelerator walls," Proc. Ecloud'12, CERN-2013-002, p 105-15 (2013).

[10] Schäfers, F. H.-Ch. Mertins, H.-Ch. Gaupp, A., Gudat, W. Mertin, M., Packe, I., Schmolla, F., DiFonzo, S., Soullie, G. Jark, W. Walker, R.P. Le Cann, X. Nyholm, R. Eriksson, M., "A Soft Soft X-Ray Polarimeter using Multilayer Optics: Complete Analysis of the Polarization State of Light," Applied Optics 38, 4074-88 (1999).

[11] Schäfers, F., Mertins, H., Schmolla, F., Packe, I., Salashchenko, N. N., Shamov, E. A., "Cr /Sc Multilayers for the Soft-X-Ray Range,” Appl. Opt. 37, 719-728 (1998).

[12] Chkhalo, N.I. Künstner, S. Polkovnikov, V.N. Salashchenko, N.N. Schäfers, F. Starikov, S.D., "High performance $\mathrm{La} / \mathrm{B}_{4} \mathrm{C}$ multilayer mirrors with C-barrier layers for the next generation lithography," Appl. Phys. Lett. 102, 011602 1-3 (2013).

[13] MacDonald, M.A., Schäfers, F. Gaupp, A., "A single W/B ${ }_{4} C$ transmission multilayer for polarization analysis of soft X-rays up to $1 \mathrm{keV}$," Optics Express 17(25), 23290-8 (2009).

[14] Gaupp, A., Schäfers, F., Uschakow, S. MacDonald, M.A., Salashchenko N.N., Gaykovich, P.K., "Carbon Kedge Polarimetry with $\mathrm{Cr} / \mathrm{Sc}$ transmission multilayers," J. Phys.: Conf. Ser. 425, 122013, 1-4 (2013).

[15] Uschakow, S. Gaupp, A. MacDonald, M.A., Schäfers, F., "EUV Ellipsometry on Mo/Si Multilayers,” J. Phys.: Conf. Series 425, 152011, 1-4 (2013).

[16] Filatova, E. O., Sokolov, A. A., Kozhevnikov, I. V., Taracheva, E. Yu., Grunsky, O. S., Schäfers, F., Braun, W., "Investigation of the structure of thin $\mathrm{HfO}_{2}$ films by soft x-ray reflectometry techniques," J. Phys.: Condens. Matter 21, 185012 (2009).

[17]Filatova, E., Taracheva, E., Shevchenko, G., Sokolov, A., Kozhevnikov, I., Yulin, S., Schäfers, F., Braun, W., "Atomic ordering in $\mathrm{TiO}_{2}$ thin films studied by X-ray reflection spectroscopy," Phys. Status Solidi B, 1-5 (2009). 
[18]Filatova, E.O., Kozhevnikov, I.V., Sokolov, A.A., Konashuk, A.S., Schäfers, F., Popovici, M., Afanas'ev, V.V., "Study of underlayer material influence on the structure of atomic-layer deposited $\mathrm{SrTi}_{\mathrm{x}} \mathrm{O}_{\mathrm{y}}$ films using soft Xray reflectometry,"J. Electron Spectrosc. Relat. Phenom., in press (2014).

[19] Filatova, E.O., Kozhevnikov, I.V., Sokolov, A.A., Ubyivovk, E.V., Yulin, S., Gorgoi, M., Schäfers, F., "Soft xray reflectometry, hard x-ray photoelectron spectroscopy and transmission electron microscopy investigations of the internal structure of $\mathrm{TiO}_{2}(\mathrm{Ti}) / \mathrm{SiO}_{2} / \mathrm{Si}$ stacks," Sci. Technol. Adv. Mater. 13, 1015001 (2012).

[20] Filatova, E.O. and Lukyanov V. A., "Anisotropy of x-ray reflectivity: chemical and structural effects on Kshell excitations in hexagonal BN crystal," J. Phys.: Condens. Matter, 14, 11643 (2002).

[21] Filatova, E.O., Taracheva, E. Yu., André, J.- M., Mertins, H.-Ch., Abramsohn, D., "Polarisation effect in the xray reflection of hexagonal BN crystal" J. Electron Spectroscopy \& Related Phenomena 144-147, 937-941 (2005).

[22] Konyushenko, M.A., Konashuk, A.S., Sokolov, A.A., Schaefers, F., Filatova, E.O., "Effect of thermal annealing and $\mathrm{Al}_{2} \mathrm{O}_{3}$-interlayer on intermixing in the $\mathrm{TiN} / \mathrm{SiO}_{2} / \mathrm{Si}$ structure," Journal of Electron Spectroscopy and Related Phenomena, in press (2014).

[23] Filatova, E., Lukyanov, V., Barchewitz, R., Andŕe, J.-M., Idir, M., Stemmler, P., "Optical constants of amorphous $\mathrm{SiO}_{2}$ for photons in the range of 60-3000 eV," J. Phys. Condens. Matter 11, 3355-3370 (1999).

[24] Filatova, E., Sokolov, A., André, J., Schäfers, F., Braun, W., "Optical constants of crystalline $\mathrm{HfO}_{2}$ for energy range 140-930 eV," Appl. Opt. 49, 2539-2546 (2010).

[25] Tümmler, J. et al., "Characterization of the PTB EUV reflectometry facility for large EUVL optical components," Proc. SPIE Vol. 5037, 265-273 (2003).

[26] Laubis, Ch. Scholze, F. Buchholz, Ch., Fischer, A., Hesse, S., Kampe, A., Puls, J., Stadelhoff, Ch. Ulm, G., "High accuracy EUV reflectometry at large optical components and oblique incidence," Proc. SPIE Vol. 7271, 1-9 (2009).

[27] Underwood, J.H., Gullikson, E.M., "High-resolution, high-flux, user friendly VLS beamline at the ALS for the 50-1300 eV energy region," J. Electron Spectrosc. Relat. Phenom. 92, 265-272 (1998).

[28]Eggenstein, F., Schäfers, F., Erko, A., Follath, R., Gaupp, A., Senf, F., Zeschke, T., "A reflectometer for atwavelength characterization of gratings," Nucl. Instrum. Meth. A 710, 166-171 (2013).

[29] Eggenstein, F., Bischoff, P. Gaupp, A., Senf, F., Sokolov, A., Zeschke, T., Schäfers, F., “A reflectometer for atwavelength characterization of XUV-reflection gratings," SPIE-Proc. $9206-7$ (2014).

[30] Follath, R., Senf, F., Gudat, W., "Plane-grating monochromator at BESSY II using collimated light," J. Synchrotron Rad. 5, 769-771 (1998).

[31] Petersen, H., Jung, C., Hellwig, C., Peatman, W. B., Gudat, W., "Review of plane grating focusing for soft xray monochromators,"Rev. Sci. Instrum. 66, 1 (1995).

[32] Riemer, F., Torge, R., "Bessy SX 700 UHV monochromator: Design features and kinematic concept,"Nuclear Instruments and Methods In Physics Research, 208, 313-314 (1983).

[33] Siewert, F., Metrology, "Mirrors and Gratings - Advances and Challenges in Synchrotron Optics," Journal of Physics: Conference Series 425, 152001 (2013).

[34] Schäfers F., RAY - the BESSY raytrace program, (In: Springer Series in Modern Optical Sciences: Modern Developments in X-Ray and Neutron Optics, eds. A. Erko, M. Idir, Th. Krist, A.G. Michette), Springer Berlin/Heidelberg, Vol. 137, 9-41 (2008).

[35] Kachel, T., "A novel principle of applying elliptically polarized dipole radiation in a SX700-type collimatedplane-grating soft X-ray beamline," to be published (2014).

[36] Schäfers, F., Krumrey, M., "REFLEC - A program to calculate VUV and soft x-ray optical elements and synchrotron radiation beamlines," Technischer Bericht, BESSY TB 201, 1-17 (1996).

[37] Frommherz, U., Raabe, J., Watts, B., Stefani, R., Ellenberger, U., "Higher Order Suppressor (HOS) for the PolLux Microspectroscope Beamline at the Swiss Light Source SLS," AIP Conf. Proc. 1234, 429 (2010).

[38] Waki I., Hirai Y., Momose A., and Hayakawa K., "Higher harmonics suppressor for soft x rays," Rev. Sci. Instrum. 60, 2072 (1989).

[39] Bulicke, P., Scholze, F., Wedowski, M., Ulm, G., Lammert, H., Reichardt, G., "Characterization of Si-mirrors for High order suppression at the PTB soft x-ray radiometry beamline," BESSY annual report 117 (1997). 